\title{
Description of Nucleon Induced Fission in Intranuclear Cascade - Multiparticle Preequilibrium - Hauser-Feshbach Approach
}

\author{
Sergey Yavshits ${ }^{1, a}$ and Oleg Grudzevich ${ }^{2}$ \\ 1 Khlopin Radium Institute,St. Petersburg, Russia \\ 2 State Technical University, Obninsk, Russia
}

\begin{abstract}
The MCFx code system for the description of neutron- and proton-induced fission of heavy nuclei in energy region $20-3000 \mathrm{MeV}$ is presented. The approach integrates the optical model for reaction crosssection calculations, intranuclear cascade for description of fast particle escape, exciton model for multiparticle emission of preequilibrium nucleons and Hauser-Feshbach calculations for statistical description of fission/evaporation competition. New results of systematic calculations of fission cross-sections, multiplicities and double-differential spectra of secondary particles for $\mathrm{Pb}-\mathrm{Cm}$ targets irradiated by neutrons and protons with energy $20-3000 \mathrm{MeV}$ are discussed.
\end{abstract}

\section{Introduction}

Further development of the MCFx system [1] was recently realized: relativistic corrections was introduced to OM calculations, the sharp cutting off energy for INC processes was replaced by smooth barrier penetration, Monte Carlo multi-particle preequilibrium model (MCP) was developed and tested [2], the new sets of STA parameters was generated and verified. The approach developed is adopted for evaluation of nuclear data for energies up to $3 \mathrm{GeV}$ as follows: yields of evaporation residuals; yields of fission products; spectra of secondary gammas, protons and neutrons, including emission from fission fragments; total, elastic and fission.

\section{Entrance channel}

New nuclear applications based mainly on reactions with intermediate energy nucleons meet needs in evaluated nuclear data on neutron- and proton-induced reactions above $20 \mathrm{MeV}$ and up to a few GeV. A scarcity of the experimental data in this energy range requires carrying out of theoretical model calculations, the most of which contains as a necessary physical component the nucleon probability of penetration into a target nucleus, i.e. the optical model reaction (absorption) cross section.

The optical potentials are key quantities in any optical model calculations and therefore their reliable definition is very important. Simulation of the entrance channel within the framework of a coupled channel method [3] in a relativistic approximation and development of a global optical potential for heavy nuclei in wide energy region were performed. The comparisons of the available experimental

\footnotetext{
a e-mail: yav@mail.rcom.ru
}

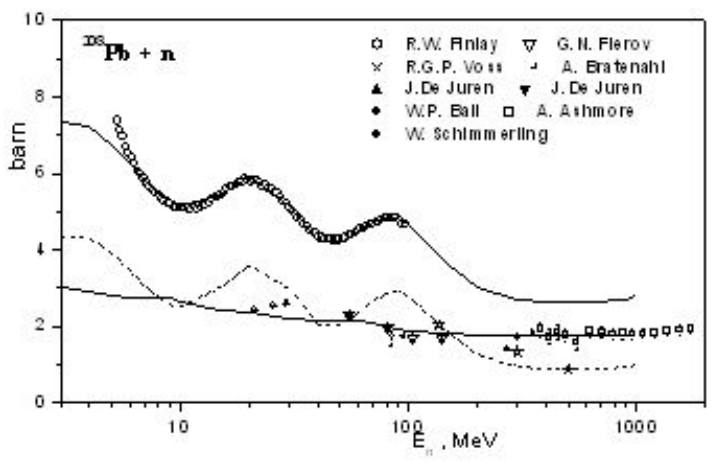

Fig. 1. Optical cross sections for interaction ${ }^{208} P b+n$. Symbols are the experimental data. The curves are total reaction cross section (top), shape elastic cross section (dashed) and absorption cross section.

and theoretical data on total and reaction cross-sections are presented.

An extension of a beam energy range up to a few $\mathrm{GeV}$ needed some modification of the KRI2000 [4] parameterization. Namely, potentials have been simplified slightly; energy dependence has been included in the spin-orbit part; the unphysical linear functions (which are convenient in a narrow energy range) have been replaced by the exponential dependences; some additional fit has been carried out to the available experimental data for the projectile energy up to $3 \mathrm{GeV}$. Note that these modifications practically have no influence on the reaction cross sections calculated for the energy region below $200 \mathrm{MeV}$.

The standard optical model of the nuclear reactions can be used up to about $200 \mathrm{MeV}$ without appreciable difficulties due to relativistic effects. The analysis of nucleon-

This is an Open Access article distributed under the terms of the Creative Commons Attribution-Noncommercial License, which permits unrestricted use, distribution, and reproduction in any noncommercial medium, provided the original work is properly cited. 


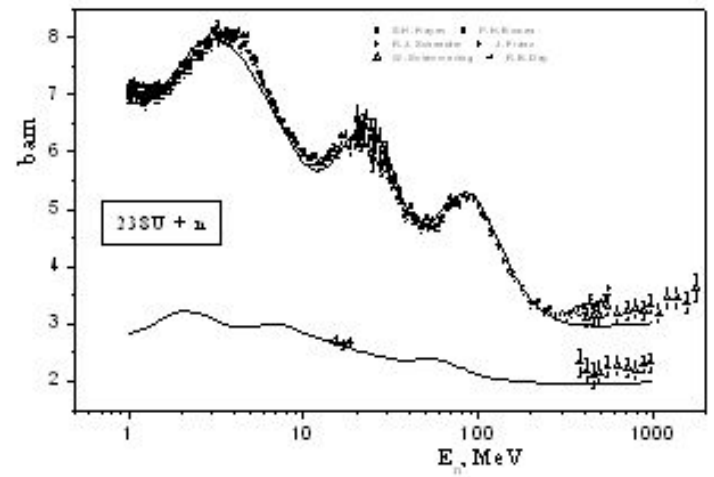

Fig. 2. Optical cross sections for interaction ${ }^{238} U+n$. Symbols are the experimental data. The curves are total reaction cross section (top) and absorption cross section.

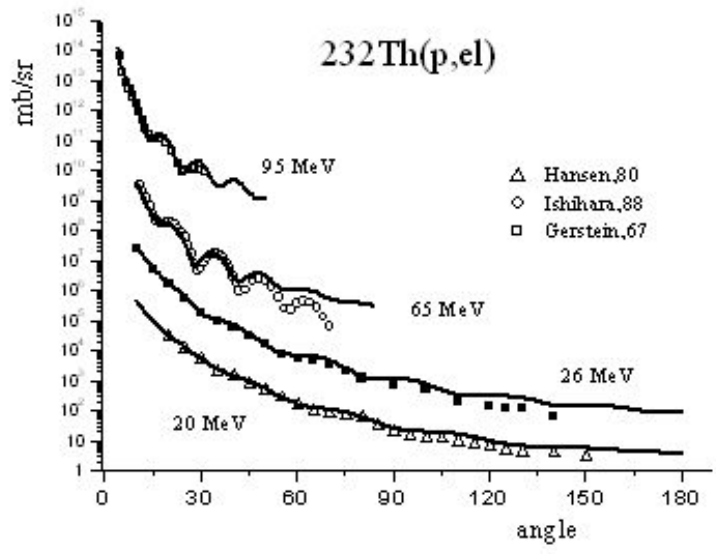

Fig. 3. Angular distributions of elastic scattering for ${ }^{232} \mathrm{Th}+\mathrm{p}$ at different projectile energies: 20, 26, 65 and $95 \mathrm{MeV}$.

nucleus interactions at relativistic energies in terms of an optical model raises the problem of the generalization of nonrelativistic formalism based on the Schrodinger equation. The account of relativistic kinematics does not cause any difficulties (it is made, in particular, in the ECIS code). We did relativistic corrections which are necessary for inclusion in usual nonrelativistic formalism of the optical model [ ]. Comparison of the experimental and calculated results are shown in fig.1-4.

The optical model parameter set KRI2004 [4] was created by the fitting to the experimental cross sections of wide target mass region (fig.1 and 2). The optical model itself guarantees good description of the nucleon elastic scattering (fig.3 and 4). Indeed, the changing of the differential cross sections is $8-10$ orders of magnitude. The calculated data differ from the measured ones considerably for the negligible values only (fig.4, $200 \mathrm{MeV}$ ).

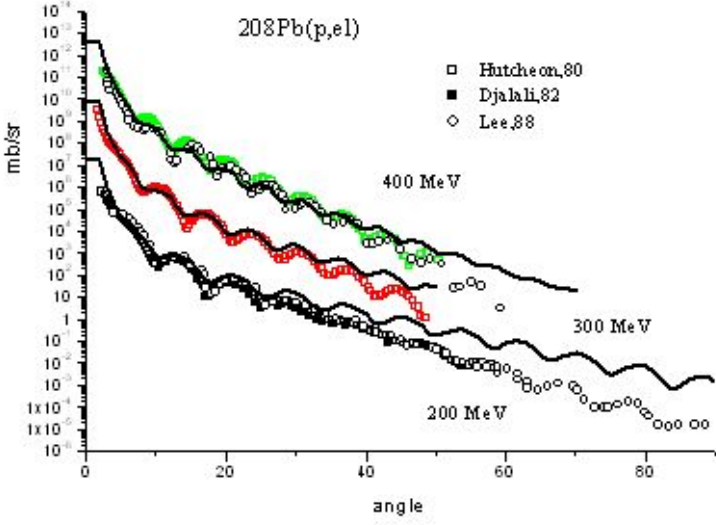

Fig. 4. Angular distributions of elastic scattering for ${ }^{208} \mathrm{~Pb}+\mathrm{p}$ at different projectile energies: 200, 300, and $400 \mathrm{MeV}$.

\section{Noncompound emission}

\section{1 intranuclear cascade}

The fast direct stage of nucleon-nucleus interaction in the MCFx code is described with the modified Dubna version of intranuclear cascade model [5]. All calculations of intranuclear cascades are performed in the three dimension geometry. The function of nuclear density distribution are described by Fermi distribution with parameters taken from the experimental data on electron-nucleus scattering. The diffuseness of nuclear density and potential edge is taken into account. For intranuclear collisions of nucleons the Pauli principle forbids the collision with energy of secondary particles less the Fermi energy.

The main condition of intranuclear cascade model applicability is the smallness of the length of de Broglie waves for all interacting particles; the wave length must be less than average distance between intranuclear nucleons 1 $\mathrm{fm}$. In this case the picture of interaction is approximately the semiclassical one and it is possible to say about the particle trajectories and two-particle collisions inside nucleus. This condition restricts the energy region of incident particles as $E \geq 50 \mathrm{MeV}$. Such a limitation is a significant point of the model. Practically the lowest limit of intranuclear cascade applicability can be established by the analysis of calculation results. We replaced the cut off threshold by the parabolic barrier with fixed height $(B \sim 10 \mathrm{MeV})$ and width $(W \sim 50 \mathrm{MeV})$. So it is smooth slowing down of an INC contribution to the reaction cross sections as a projectile energy decreases.

\subsection{Preequilibrium multiparticle emission}

The idea of multiparticle preequilibrium emission in the frameworks exciton statistical model was proposed a long time ago [6] but as it is known to authors was realized recently. Its meaning is clear - after the first nucleon emission to repeat the calculation of equilibration for new composite 


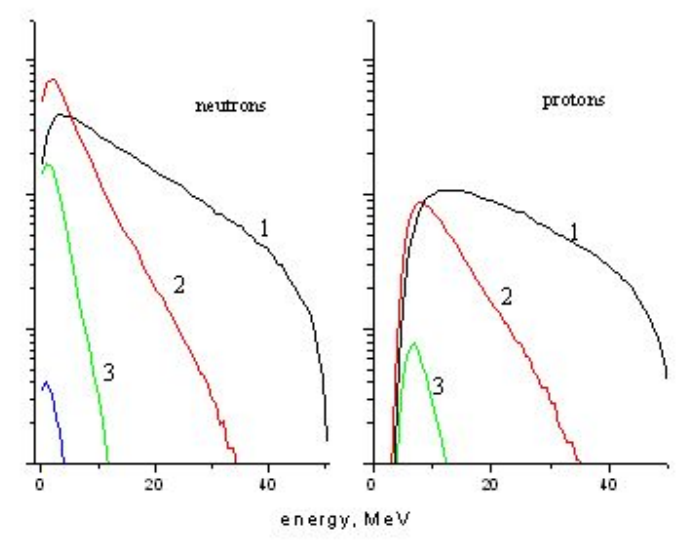

Fig. 5. Neutron (left) and proton (right) spectra of preequilibnrium decay of ${ }^{93} \mathrm{Nb}+\mathrm{n}$ composite system at $50 \mathrm{MeV}$ projectile energy. Nucleon orders are marked.

system etc. In the proposed scheme of calculation (model MCP) the reaction cross sections for nucleons of the intermediate and high energies at a preequilibrium stage which begins with various initial excitations and configurations, residual nucleus should pass to equilibrium emitting out the nucleons.

At the first stage of calculations according to initial excitation energy and initial quasi-particle configuration ph of the first nucleus the list of nucleus which may be formed as a result of nucleon emission and their excitation energies $\mathrm{E}^{\text {max }}$ is determined.

At the second stage of calculations for all possible nuclei and their excitation energies from 0 up to $E^{\max }$ the probabilities of the nucleon emission and the quasiparticle transition probabilities are calculated. The lists of the particle-hole configurations are formed so that the number of particles in the first configuration differed from the previous nucleus by the number of emitted nucleons. The further stages are carried out the given number of times (histories of the Monte Carlo simulating).

The system of the master-equations is solved (stage 3 ). The probabilities are chosen by a random number usage to define the type of process: quasiparticle transition; neutron / proton emission. After the quasiparticle transition the solution of the master-equation system is prolonged i.e the composite system moves to equilibrium. After emission of a nucleon its energy is defined by a random number according to the low of a energy distribution. The emission of the nucleon leads to decrease of excitation energy of composite system as well as decrease of $(\mathrm{Z}, \mathrm{A})$ values and decrease of number of quasi-particles by 1 unit. The values obtained are used as initial quantities for the following calculations. Transition to a stage 3 is carried out further and the master-equation system for new starting conditions is solved.

The history comes to the end if the state of equilibrium is achieved or the energy of a residual nucleus is insufficient for the nucleon emission. The nucleus population and particle spectra are stored.

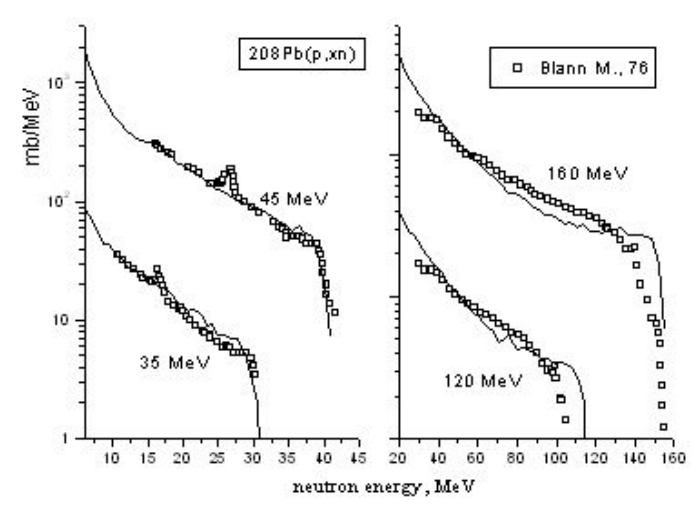

Fig. 6. Neutron energy distributions of ${ }^{208} \mathrm{~Pb}(\mathrm{p}, \mathrm{xn})$ reaction. Projectile energies are marked. The symbols are experimental data [7].

The nucleon spectra of composite system preequilibrium decay is shown in fig.5. It is seen that as the number of nucleon increases as the composite system goes to equilibrium - the spectrum shape becomes softer and it looks like an evaporated one. One problem exists in this situation: in what time moment can one believes the system is at equilibrium? We fixed the maximal number of preequilibrium neutrons $N_{n}^{\max }=5$ and $N_{p}^{\max }=2$. If we are wrong the undertaken preequilibrium (but soft!) nucleons will be evaporated by the compound system. Obviously these values have to be increased for a few $\mathrm{GeV}$ projectile energies.

\subsection{Stage result fusion}

The fist stage of calculations with MCFx code was intranuclear cascade block. The results of this stage are the energy distributions (spectra) of the nucleons $S_{I N C}(\varepsilon)$ the residual nucleus yields $Y_{I N C}(Z A, E, p h)$ as the function of excitation energy and particle-hole configuration. The data on particle spectra are used to final spectra modeling. The $Y_{I N C}$ values are used as the input data for MCP preequilibrium model calculations. The results of this model are nucleon spectra $S_{M C P}(Z A, E, p h, \varepsilon)$ and the distributions of the residual nuclei at the equilibrium states $Y_{M C P}(Z A, E)$. The $Y_{M C P}$ values are the part of input data for the statistical model calculations. Other part of the input data is typical statistical model input quantities: level density parameters, discrete level information, transmission coefficients, fission barrier parameters. After the usage of Hauser-Feshbach formalism we have got a lot of information: statistical spectra $S_{H F}(Z A, E, \varepsilon)$; ground state nuclear distributions, fission cross sections etc..

The calculated final spectrum of escaped particles is the sum of all reaction mechanism as:

$$
\begin{gathered}
S(\varepsilon)=S_{I N C}(\varepsilon)+ \\
+\sum \sum \sum Y_{I N C}(Z A, E, p h) \times S_{M C P}(Z A, E, p h, \varepsilon)+
\end{gathered}
$$




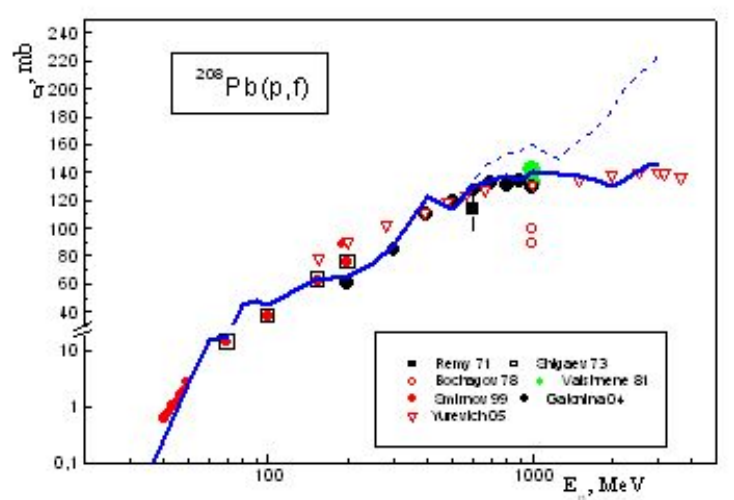

Fig. 7. Fission cross section of ${ }^{208} \mathrm{~Pb}+\mathrm{p}$ reaction. The symbols are exerimental data. The dashed curve shows unfitted calculation results, solid line presents the results of fitting by fission barrier changing.

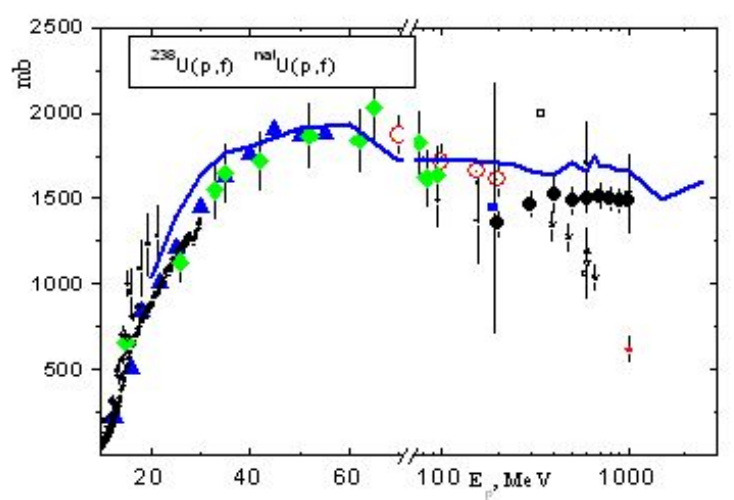

Fig. 8. Fission cross section of ${ }^{238} U+p$ reaction. The symbols are exerimental data. The curve shows calculation results. No fitting was done.

$$
+\sum \sum Y_{M C P}(Z A, E) \times S_{H F}(Z A, E, \varepsilon)
$$

The summing in (1) has to be done for all nuclei, all excitation energies and all configuratons. The results of neutron spectrum calculations and the experimental data on the ${ }^{208} \mathrm{~Pb}(\mathrm{p}, \mathrm{xn})$ reaction are shown in fig.6 for different projectile energies. One can see that the agreements are good for all cases. It means the combination of the three mechanism contributions was done correctly:

\section{Fission cross sections}

The fission cross sections of the two different types of fissile nuclei are shown in fig. 7 and 8 . The ${ }^{235} \mathrm{U}$ has high fission probabilities. On the other hand, the fission cross sections of ${ }^{208} \mathrm{~Pb}$ are small (in comparison with the reaction cross section) for the projectile energies up to $1 \mathrm{GeV}$. It is known the description of small cross sections, which are very sensitive on the competition of more strong reaction channels, is enough complicated. On the another hand, the fitting can be done easily by the fission barrier changing. The results presented in figs. 7 and 8 were calculated without the parameter fitting. In fact, the data comparison shows the real predictability of MCFx code. It can be seen that for ${ }^{238} \mathrm{U}$ target the experimental fission cross sections, in spite of some deviation, are described by the theoretical calculations reasonably good for proton energies from 20 $\mathrm{MeV}$ to $1000 \mathrm{MeV}$. On the contrary, the measured fission cross sections of ${ }^{208} \mathrm{~Pb}$ target are worse described by the calculations especially for proton energies from $700 \mathrm{MeV}$ to $3000 \mathrm{MeV}$. The main reason of this bad description is small values of cross sections.

\section{Conclusion}

The results of nucleon emission characteristics and fission cross sections calculations are presented and discussed. The problems of the composite system movement to equilibrium are mentioned.

The work was performed under ISTC Project 3751.

\section{References}

1. Yavshits S. et al. In Proc. of the Int. Conf. on Nucl. Data for Sci. and Tech., Tsukuba, Japan, (2001) pp. 104-107 .

2. Grudzevich O. et al. Oxford University Press, Radiation Protection Dosimetry, Volume 26, 1-4, (2007), 101-103.

3. J. Raynal, Notes on ECIS94, CEA Saclay Report No. CEA-N-2772, 1994.

4. S.Yavshits, G.Boykov, et al., Proc. of the 9 th International Conference on Nuclear Reaction Mechanisms, ed. by E. Gadioli, Varenna, June 5-9, 2000, p. 219.

5. K.K. Gudima, S.G. Mashnik, V.D. Toneev, Nucl.Phys., Volume A401, (1983) 329.

6. Akkermans J.M. and Gruppelaar H., Z. Phys., Volume A300, (1981), p.345.

7. Blann M., e.a., Nucl. Phys., Volume A257, (1976), p. 15 . 
This page is available in the following languages

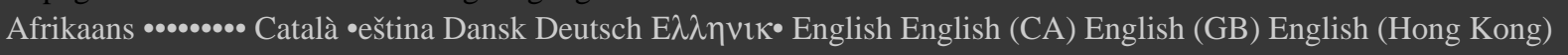
English (Singapore) English (US) Esperanto Castellano Castellano (AR) Español (CL) Castellano (CO) Español

(Ecuador) Español (Guatemala) Castellano (MX) Castellano (PE) Euskara Suomeksi français français (CA) Galego •••• hrvatski Magyar Italiano ••• •• Macedonian Melayu Nederlands Norsk Sesotho sa Leboa polski Português român• slovenski jezik $\bullet \bullet \bullet \bullet$ srpski (latinica) Sotho svenska $\bullet \bullet \bullet \bullet \bullet \bullet \bullet \bullet \bullet(\bullet)$ isiZulu

\section{(c) \\ (c) commons}

\section{Attribution-Noncommercial 3.0 Unported}

\section{You are free:}

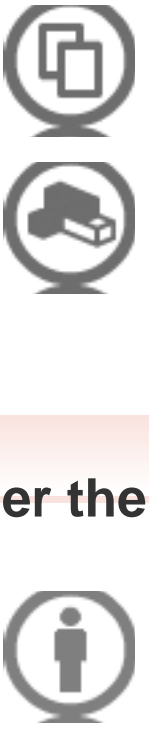

- to Share - to copy, distribute and transmit the work

- to Remix — to adapt the work

\section{Under the following conditions:}

- Attribution - You must attribute the work in the manner specified by the author or licensor (but not in any way that suggests that they endorse you or your use of the work).

- Noncommercial - You may not use this work for commercial purposes.

\section{With the understanding that:}

- Waiver - Any of the above conditions can be waived if you get permission from the copyright holder.

- Public Domain - Where the work or any of its elements is in the public domain under applicable law, that status is in no way affected by the license. 
- Other Rights - In no way are any of the following rights affected by the license:

- Your fair dealing or fair use rights, or other applicable copyright exceptions and limitations;

o The author's moral rights;

- Rights other persons may have either in the work itself or in how the work is used, such as publicity or privacy rights.

- Notice - For any reuse or distribution, you must make clear to others the license terms of this work. The best way to do this is with a link to this web page.

\section{Disclaimer}

The Commons Deed is not a license. It is simply a handy reference for understanding the Legal Code (the full license) - it is a human-readable expression of some of its key terms. Think of it as the user-friendly interface to the Legal Code beneath. This Deed itself has no legal value, and its contents do not appear in the actual license.

Creative Commons is not a law firm and does not provide legal services. Distributing of, displaying of, or linking to this Commons Deed does not create an attorney-client relationship. 